\title{
Analysis of TOF-SIMS spectra using quaternary ammonium ions for mass scale calibration
}

\author{
Daisuke Kobayashi ${ }^{* 1}$, Shinya Otomo ${ }^{2}$ and Hiroto Itoh $^{3}$ \\ ${ }^{1}$ Research Center, ASAHI GLASS CO., LTD., 1150 Hazawa-cho, Kanagawa-ku, Yokohama, Kanagawa 221-8755 Japan \\ ${ }^{2}$ Yokohama R\&D Laboratories, Furukawa Electric CO., LTD., 2-4-3 Okano, Nishi-ku, Yokohama 220-0073 Japan \\ ${ }^{3}$ Material Analysis Technology Division, KONICAMINOLTA, INC., 1 Sakura-machi, Hino-shi, Tokyo 191-8511 Japan \\ *daisuke-kobayashi@agc.com
}

(Received : November 27, 2013; Accepted : February 11, 2014)

\begin{abstract}
A novel method with quaternary ammonium salts as internal additives has been applied to the mass scale calibration of time-of-flight secondary ion mass spectrometry (TOF-SIMS). Five kinds of quaternary ammonium salts, octyltrimethylammonium bromide (C8TMA), tetradecyltrimethylammonium chloride (C14TMA), octadecytrimethylammonium chloride (C18TMA), cetylpyridinium chloride (CPC) and benzyldimethyltetradecylammonium chloride (Bzl) were diluted with distilled water and conc- $\mathrm{NH}_{3}$ and were mixed in equal quantities. The solution was added to Tinuvin 770 coated on a Si wafer. Then, the sample was measured by TOF-SIMS. The relation between the relative mass accuracy of molecular ions of Tinuvin 770 and the combinations of ions selected for the mass scale calibration was investigated. To improve the mass accuracy of the molecular ion of Tinuvin 770, ions should be selected for the mass scale calibration in the following two ways: one way is for ions to consist only of molecular ions of C8TMA, C14TMA and C18TMA, and the other way is for ions to include the molecular ion of C18TMA, along with the $\mathrm{C}_{X} \mathrm{H}_{Y}$ fragment ions. These results support the proposition of ISO 13084. The novel method with internal additives is effective to improve the mass accuracy of high-mass ions. Quaternary ammonium salts are potential candidates of internal additives.
\end{abstract}

\section{Introduction}

Time-of-flight secondary ion mass spectrometry (TOF-SIMS) has been recognized as one of the most important surface analysis techniques. TOF-SIMS has features of high mass resolution and wide mass range. To extract information from the TOF-SIMS spectrum, horizontal scale of the spectrum should change from time-of-flight to mass by mass scale calibration. A conventional method for the mass scale calibration is conducted by extrapolation of several identified secondary ions with low mass number [1, 2]. However, identifying an unknown high-mass peak in the spectrum is difficult because an accurate mass scale of the spectrum is not obtained by the conventional method [3]. In 2011, ISO 13084 was formulated in terms of a procedure for the mass scale calibration. According to the ISO 13084, the mass scale calibration should be carried out by the use of some ions with less degradation or fragmentation from the original parent structure. In addition, to identify molecules with a larger mass $m$, the inclusion of an ion with a mass $m_{A}$ which satisfies $m_{A} \geq 0.55 m$ is highly recommended for the mass scale calibration. The effectiveness of ISO 13084 was confirmed by an interlaboratory study [4]. It is, however, general that TOF-SIMS spectra do not contain correctly identified ions which satisfy $m_{A} \geq 0.55 m$.

A practical method for mass scale calibration in TOF-SIMS has been discussed by the TOF-SIMS working group (TOF-SIMS WG) which was established in Surface Analysis Society of Japan in June 2007 [5 9]. One of the objectives of TOF-SIMS WG is to figure out a novel mass scale calibration for improvement of the mass accuracy of a high-mass ion. A novel method with quaternary ammonium salts as internal additives has been proposed [9]. ISO 13084 recommendations are met by selection of molecular ions of quaternary ammonium 

bration

salts for mass scale calibration. The novel method is expected to provide more accurate mass scale than the conventional one. In this study, Tinuvin $770\left(\mathrm{C}_{28} \mathrm{H}_{52} \mathrm{~N}_{2} \mathrm{O}_{4}\right.$, molecular weight $480.39 \mathrm{u}$ ), which is a popular UV stabilizer, is used as the target sample. The effectiveness of the novel method is evaluated by the use of Tinuvin 770 coated on a Si wafer. The relation between the relative mass accuracy of molecular ions of Tinuvin 770 and the combinations of ions selected for mass scale calibration is investigated.

\section{Experimental}

Approximately a $10 \mathrm{mg}$ granule of Tinuvin 770 was dissolved into $1 \mathrm{ml}$ of acetone and was coated on a hydrophilic-treated Si wafer by a spin-coater (3000 rpm, 1 min). Five kinds of quaternary ammonium salts with different molecular weights were prepared as internal additives: octyltrimethylammonium bromide (C8TMA), tetradecyltrimethylammonium chloride (C14TMA), octadecytrimethylammonium chloride (C18TMA), cetylpyridinium chloride (CPC) and benzyldimethyltetradecylammonium chloride (Bzl). These quaternary ammonium salts were diluted with $10 \mathrm{ml}$ of distilled water and $20 \mu \mathrm{l}$ of conc- $\mathrm{NH}_{3}$ to approximately $1 \mathrm{mmol} / \mathrm{l}$, respectively, and were mixed in equal quantities. The mixed solution was the same as the one used in our previous reports [7, 9]. Several drops of the mixed solution added to Tinuvin 770 coated on a Si wafer by a dropper.

The mass spectra of the sample were obtained by a TOF-SIMS 5 (ION-TOF, GmbH) with a single-stage reflection design [10]. $\mathrm{Bi}_{3}{ }^{++}$was used as a primary ion at $25 \mathrm{kV}$ with a pulse width of $4.3 \mathrm{~ns}$ before bunching. The relevant settings were: cycle time $150 \mu \mathrm{s}$, and region of interest $200 \times 200 \mu \mathrm{m}^{2}$. Energy of analyzer was set at $-1000 \mathrm{~V}$. It was confirmed that intensity of secondary ions are not saturated under these conditions. The electron shower for positive charge compensation was not used in this work. Four different positions on the Si wafer were measured to confirm repeatability of the relative mass accuracy of secondary ions.

\section{Results and discussion}

Figure 1 shows positive TOF-SIMS spectra of Tinuvin 770 coated on a Si wafer with and without internal additives. A molecular ion derived from Tinuvin 770 was observed as $\mathrm{C}_{28} \mathrm{H}_{53} \mathrm{~N}_{2} \mathrm{O}_{4}{ }^{+}$by addition of a hydrogen ion. In the case of with internal additives, not only the molecular ion of Tinuvin 770, but also molecular ions of

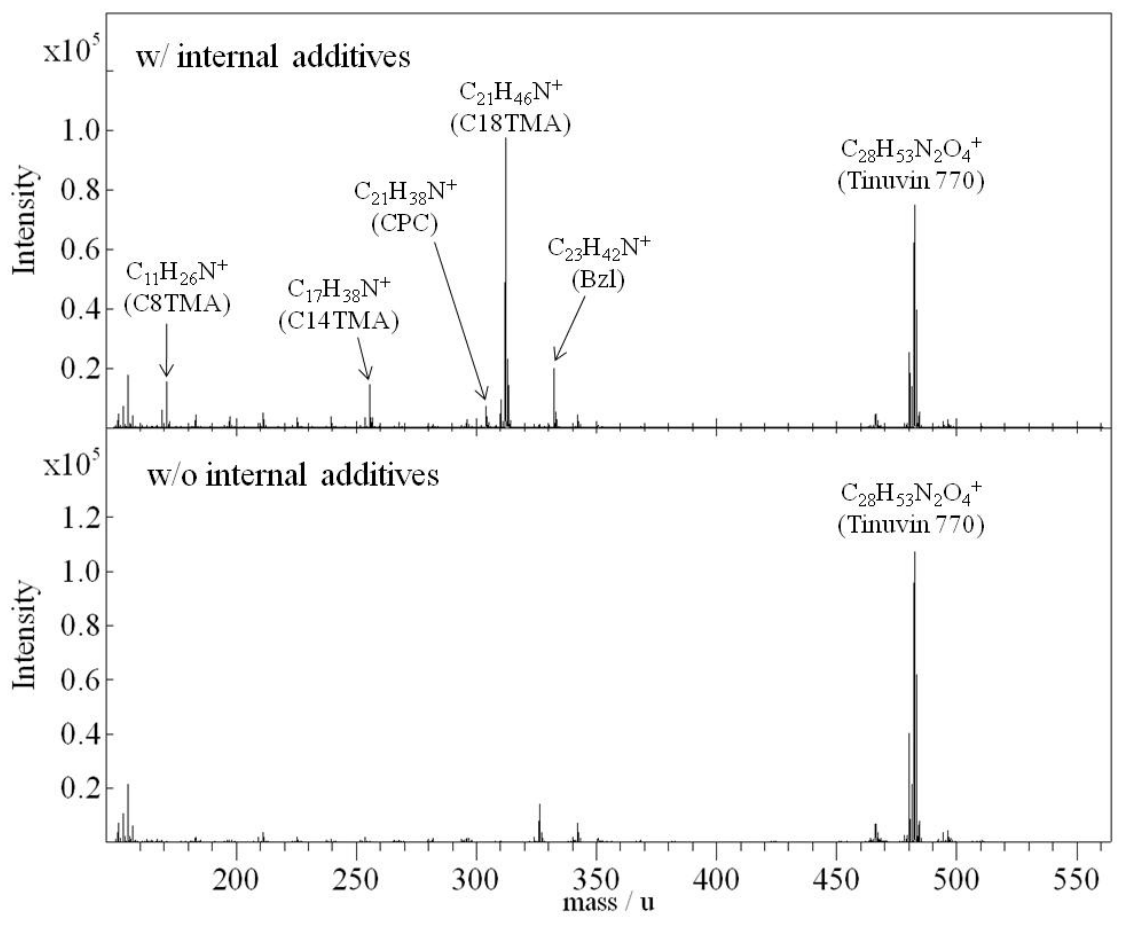

Fig. 1 Positive TOF-SIMS spectra of Tinuvin 770 coated on the Si wafer with (upper) and without (lower) internal additives. 

bration

quaternary ammonium salts were clearly detected: $\mathrm{C}_{11} \mathrm{H}_{26} \mathrm{~N}^{+}$derived from C8TMA, $\mathrm{C}_{17} \mathrm{H}_{38} \mathrm{~N}^{+}$derived from C14TMA, $\mathrm{C}_{21} \mathrm{H}_{38} \mathrm{~N}^{+}$derived from CPC, $\mathrm{C}_{21} \mathrm{H}_{46} \mathrm{~N}^{+}$derived from C18TMA, and $\mathrm{C}_{23} \mathrm{H}_{42} \mathrm{~N}^{+}$derived from Bzl. C8TMA, C14TMA and C18TMA are alkali quaternary ammonium salts and have similar chemical structures. Therefore, molecular ions of these alkali quaternary ammonium salts were actively selected for mass scale calibration in this study.

Along the lines of ISO 13084, we defined the mass accuracy, $\Delta M$, as the difference between the measured peak mass, $M_{p}$, and the true mass, $M_{T}$, given by:

$$
\Delta M=M_{P}-M_{T}
$$

and the relative mass accuracy, $W$, when expressed numerically in parts-per-million by:

$$
W=\Delta M / M_{T} \times 10^{6}
$$

First, a mass scale calibration was conducted by $\mathrm{C}_{\mathrm{X}} \mathrm{H}_{\mathrm{Y}}$ fragment ions or molecular ions. Table 1 shows combinations of ions selected for mass scale calibration, which consist only of $\mathrm{C}_{X} \mathrm{H}_{Y}$ fragment ions or molecular ions derived from internal additives. Combinations $\mathrm{A}$ and $\mathrm{B}$ have been selected by interlaboratory tests among TOF-SIMS WG $[5,8]$. Combination C consists of the $\mathrm{C}_{\mathrm{X}} \mathrm{H}_{\mathrm{Y}}$ fragment ions with degree of unsaturation, $D U, 1.5$ and has been selected by our previous report [7, 9]. The degree of unsaturation of $\mathrm{C}_{\mathrm{X}} \mathrm{H}_{\mathrm{Y}}$ fragment ion is the index of hydrogen deficiency and is given by:

$$
D U=1+X-Y / 2
$$

$X$ and $Y$ are the number of carbon atoms and hydrogen atoms, respectively. Combination D consists of molecular ions of C8TMA, C14TMA and C18TMA. Fig. 2

Table 1 Combinations of ions selected for mass scale calibration, which consist only of $\mathrm{C}_{\mathrm{X}} \mathrm{H}_{\mathrm{Y}}$ fragment ions or molecular ions derived from internal additives.

\begin{tabular}{cl}
\hline Combination & Ions selected for mass scale calibration \\
\hline \hline A & $\mathrm{CH}_{3}, \mathrm{C}_{2} \mathrm{H}_{3}, \mathrm{C}_{3} \mathrm{H}_{5}$ \\
B & C, $\mathrm{CH}, \mathrm{CH}_{2}, \mathrm{C}_{4} \mathrm{H}_{3}, \mathrm{C}_{4} \mathrm{H}_{5}$ \\
C & CH, $\mathrm{C}_{2} \mathrm{H}_{3}, \mathrm{C}_{3} \mathrm{H}_{5}, \mathrm{C}_{4} \mathrm{H}_{7}, \mathrm{C}_{5} \mathrm{H}_{9}$ \\
D & C8TMA, C14TMA, C18TMA \\
\hline
\end{tabular}

shows the difference of relative mass accuracy of Tinuvin 770 among combinations A $\sim$ D. The relative mass accuracy was confirmed by four repeat measurements at different measurement points. The repeatability is ensured to discuss on the difference among combinations. The relative mass accuracy of Tinuvin 770 with combination B is better than with combinations A and C. This trend is inconsequential considering the distribution of relative mass accuracy of $\mathrm{C}_{\mathrm{X}} \mathrm{H}_{\mathrm{Y}}$ fragment ions, as shown in previous report $[3,9]$. On the other hand, the relative mass accuracy of Tinuvin 770 is improved by combination $\mathrm{D}$ and indicates approximately +11 ppm on average. When molecular ions of C8TMA, C14TMA and C18TMA were used for the mass scale calibration, the relative mass accuracy of low-mass $\mathrm{C}_{X} \mathrm{H}_{Y}$ fragment ions indicated negative values [7,9]. Considering the collision cascade model of the static SIMS, $\mathrm{C}_{\mathrm{X}} \mathrm{H}_{\mathrm{Y}}$ fragment ions are expected to be generated at the center of the primary ion bombardment and to have higher kinetic energy than molecular ions $[11,12]$. When secondary ion has high kinetic energy, the relative mass accuracy of the secondary ion indicates negative values. Therefore, the mass scale calibration with low-mass $\mathrm{C}_{\mathrm{X}} \mathrm{H}_{\mathrm{Y}}$ fragment ions leads to the degradation of the relative mass accuracy of high-mass molecular ions. Combination D which consists only of molecular ions improves the relative mass accuracy of Tinuvin 770.

Second, mass scale calibration was conducted by $\mathrm{C}_{\mathrm{X}} \mathrm{H}_{\mathrm{Y}}$ fragment ions and one of the molecular ions derived from internal additives. Table 2 shows these combinations and $m_{A} / m$. Combinations A' $\sim$ C', A'” $\sim$ C', and A"” C", include one of the molecular ions of C8TMA,

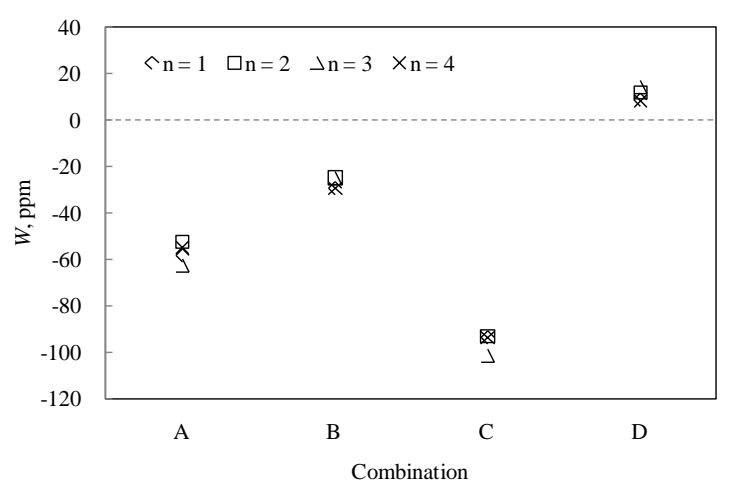

Fig. 2 Difference of relative mass accuracy, $W$, of Tinuvin 770 among combinations A D. Values of relative mass accuracy were confirmed by four repeat measurements at different measurement points. 

bration

Table 2 Combinations of ions selected for mass scale calibration, which consist of $\mathrm{C}_{X} \mathrm{H}_{\mathrm{Y}}$ fragment ions and one of the molecular ions derived from internal additives, and $m_{A} / m$.

\begin{tabular}{|c|c|c|}
\hline Combination & Ions selected for mass scale calibration & $m_{A} / m$ \\
\hline$\overline{A^{\prime}}$ & $\mathrm{CH}_{3}, \mathrm{C}_{2} \mathrm{H}_{3}, \mathrm{C}_{3} \mathrm{H}_{5}, \mathrm{C} 8 \mathrm{TMA}$ & 0.36 \\
\hline $\mathrm{B}^{\prime}$ & C, $\mathrm{CH}, \mathrm{CH}_{2}, \mathrm{C}_{4} \mathrm{H}_{3}, \mathrm{C}_{4} \mathrm{H}_{5}$, C8TMA & 0.36 \\
\hline $\mathrm{C}^{\prime}$ & $\mathrm{CH}, \mathrm{C}_{2} \mathrm{H}_{3}, \mathrm{C}_{3} \mathrm{H}_{5}, \mathrm{C}_{4} \mathrm{H}_{7}, \mathrm{C}_{5} \mathrm{H}_{9}$, C8TMA & 0.36 \\
\hline $\mathrm{A}^{\prime \prime}$ & $\mathrm{CH}_{3}, \mathrm{C}_{2} \mathrm{H}_{3}, \mathrm{C}_{3} \mathrm{H}_{5}, \mathrm{C} 14 \mathrm{TMA}$ & 0.53 \\
\hline B" & C, $\mathrm{CH}, \mathrm{CH}_{2}, \mathrm{C}_{4} \mathrm{H}_{3}, \mathrm{C}_{4} \mathrm{H}_{5}$, C14TMA & 0.53 \\
\hline $\mathrm{C}^{\prime \prime}$ & $\mathrm{CH}, \mathrm{C}_{2} \mathrm{H}_{3}, \mathrm{C}_{3} \mathrm{H}_{5}, \mathrm{C}_{4} \mathrm{H}_{7}, \mathrm{C}_{5} \mathrm{H}_{9}, \mathrm{C} 14 \mathrm{TMA}$ & 0.53 \\
\hline A"' & $\mathrm{CH}_{3}, \mathrm{C}_{2} \mathrm{H}_{3}, \mathrm{C}_{3} \mathrm{H}_{5}, \mathrm{C} 18 \mathrm{TMA}$ & 0.65 \\
\hline B"' & C, $\mathrm{CH}, \mathrm{CH}_{2}, \mathrm{C}_{4} \mathrm{H}_{3}, \mathrm{C}_{4} \mathrm{H}_{5}$, C18TMA & 0.65 \\
\hline $\mathrm{C}^{\prime \prime \prime}$ & $\mathrm{CH}, \mathrm{C}_{2} \mathrm{H}_{3}, \mathrm{C}_{3} \mathrm{H}_{5}, \mathrm{C}_{4} \mathrm{H}_{7}, \mathrm{C}_{5} \mathrm{H}_{9}$, C18TMA & 0.65 \\
\hline
\end{tabular}

C14TMA and C18TMA, respectively, along with $\mathrm{C}_{X} \mathrm{H}_{Y}$ fragment ions. Figure 3 shows the difference of relative mass accuracy of Tinuvin 770 among combinations A' C', A" C" and A'” C",'. Relative mass accuracy of Tinuvin 770 is converged by addition of one of the molecular ions, in comparison with combination $\mathrm{A} \sim \mathrm{C}$ as shown in Fig. 2. Relative mass accuracy of Tinuvin 770 is improved with high $m_{A} / m$ values. Combinations A"” C", have $m_{A} / m$ values which satisfy the ISO recommendation and provide the best relative mass accuracy, approximately $-6 \mathrm{ppm}$ on average of combinations $\mathrm{A}^{\text {’” } ~}$ C', .

Several problems still remain unsolved for applying the mass scale calibration with internal additives to a practical analysis. Since quaternary ammonium ions used as internal additives in this study are surfactant agent, there is concern that the target material is removed or changed by internal additives. Therefore, both TOF-SIMS measurements with and without internal additives are required to confirm that the target peak remains unchanged, as previously shown by Fig. 1. One of the other ways to identify an unknown target peak at high mass region is an iterative mass scale calibration which starts with identifying a particular low-mass peak. It is often possible to progressively identify peaks higher up the mass scale until the condition for $m_{A} \geq 0.55 \mathrm{~m}$ is fulfilled. When internal additives are not available, this way may be useful.

\section{Conclusion}

Relative mass accuracy of the molecular ion of

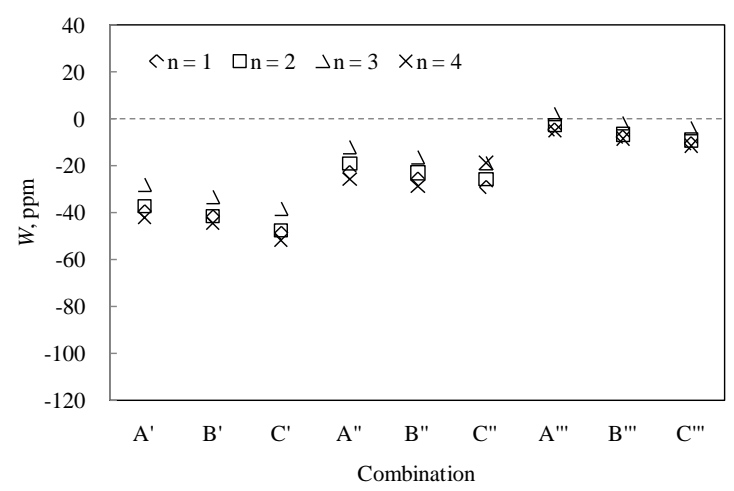

Fig. 3 Difference of relative mass accuracy, $W$, of Tinuvin 770 among combinations A' C', A" C" and A'” C",'. Values of relative mass accuracy were confirmed by four repeat measurements at different measurement points.

Tinuvin 770 is improved by the novel method with quaternary ammonium salts as internal additives. To improve the mass accuracy of the molecular ion of Tinuvin 770, ions should be selected for the mass scale calibration in the following two ways: one way is for ions to consist only of molecular ions of C8TMA, C14TMA and C18TMA, and the other way is for ions include the molecular ion of C18TMA which satisfy $m_{A} \geq 0.55 \mathrm{~m}$, along with the $\mathrm{C}_{\mathrm{X}} \mathrm{H}_{\mathrm{Y}}$ fragment ions. These results support the proposition of ISO 13084. In conclusion, the novel method with internal additives is more effective to improve the mass accuracy of high-mass ions than the conventional one. Quaternary ammonium salts are potential candidates of internal additives.

\section{References}

[1] E. Niehuis, P. N. T. Van Velzen, J. Lub, T. Heller, A. Benninghoven, Surf. Interface Anal. 14 , 135. (1989).

[2] S. Reichlmaier, J. S. Hammond, M. J. Hearn, D. Briggs, Surf. Interface Anal. 21 , 739 (1994).

[3] I. S. Gilmore, F. M. Green and M. P. Seah, Surf. Interface Anal. 39 , 817 (2007).

[4] F. M. Green, I. S. Gilmore, J. L. S. Lee, S. J. Spencer and M. P. Seah, Surf. Interface Anal. 42,129 (2010).

[5] Y. Abe, H. Itoh, S. Otomo and TOF-SIMS Working Group, J. Surf. Anal. 17 , 186 (2010).

[6] S. Otomo, H. Itoh and Y. Abe, $18^{\text {th }}$ International Conference on Secondary Ion Mass Spectrometry 280 (2011). 
Journal of Surface Analysis Vol.20, No. 3 (2014) pp. 187-191

D. Kobayashi et al. Analysis of TOF-SIMS spectra using quaternary ammonium ions for mass scale calibration

[7] D. Kobayashi, S. Aoyagi, S. Otomo and H. Itoh, The Scientific International Symposium on SIMS and Related Techniques Based on Ion-Solid Interactions 37 (2013).

[8] H. Itoh, $19^{\text {th }}$ International Conference on Secondary Ion Mass Spectrometry 357 (2013).

[9] D. Kobayashi, S. Aoyagi, S. Otomo and H. Itoh, $19^{\text {th }}$ International Conference on Secondary Ion Mass
Spectrometry 358 (2013).

[10] J. Schwieters, H. G. Cramer, T. Heller, U. Jürgens, E. Niehuis, J. Zehnphenning, A. Benninghoven, $J$. Vac. Sci. Technol. A 9, 2864 (1991).

[11] P. Bertrand, L. T. Weng, Mikrochim. Acta, Suppl. 13, 167 (1996).

[12] T. Nakanaga, H. Nagai, N. Saito, Y. Fujiwara and H. Nonaka, Int. J. Mass Spectrom. 311, 24 (2012). 